Chirurg 2022 · 93:113-114

https://doi.org/10.1007/s00104-021-01542-2

Angenommen: 5. November 2021

(C) The Author(s), under exclusive licence to Springer Medizin Verlag GmbH, ein Teil von Springer Nature 2021

Redaktion

C.-T. Germer, Würzburg

\section{Komplette Response nach neoadjuvanter Therapie}

\section{C.-T. Germer}

Klinik für Allgemein-, Viszeral-, Transplantations-, Gefäß- und Kinderchirurgie, Universitätsklinikum Würzburg, Würzburg, Deutschland
Neoadjuvante Therapiekonzepte in Form von Chemo- und/oder Strahlentherapie zur Behandlung fortgeschrittener gastrointestinaler Karzinome stellen für eine Vielzahl von Tumorentitäten den derzeitigen Behandlungsstandard dar [1, 4-6, 9]. Ziel dieser multimodalen Behandlungskonzepte ist eine Reduzierung des Lokalrezidivrisikos und häufig gleichzeitig die Verbesserung der Prognose der betroffenen Patienten. Für die meisten neoadjuvant behandelten Tumoren bleibt trotz guten Therapieansprechens die häufig mit einer kompletten Organentfernung oder zumindest einer Teilresektion einhergehende Operation der zentrale Baustein in der Behandlung bei kurativem Therapieziel.

\section{\) Organerhalt und „TME-free- Survival" sind neue Therapieziele}

Neue Behandlungskonzepte verlagern aufgrund der besseren Verträglichkeit und der höheren Patientencompliance die gesamte perioperative Behandlung vor den chirurgischen Eingriff. So wird unter dem Begriff der total neoadjuvanten Therapie (TNT) in der Behandlung des lokal fortgeschrittenen Rektumkarzinoms die Ergänzung einer präoperativen Radio- oder Radiochemotherapie um eine zusätzliche präoperative Systemtherapie (Chemotherapie) verstanden. Die bisher publizierten Daten zum Einsatz einer TNT in der Behandlung des lokal fortgeschrittenen Rektumkarzinoms zeigen, dass dieses Konzept im Vergleich zur bisherigen neoadjuvanten Therapie zu einer Erhöhung der Rate an klinischer Komplettremission führt $[2,3]$.

Vielfach wird deshalb schon als Therapieziel in der Behandlung des Rektumkarzinoms der Organerhalt und das „TME(totale mesorektal Exzision)-freeSurvival" propagiert bzw. als neues Studienziel formuliert. In der aktuell gültigen Version der S3-Leitlinie zur Behandlung des kolorektalen Karzinoms wird formuliert, dass wenn nach neoadjuvanter Therapie klinisch, endoskopisch und durch bildgebende Verfahren kein Tumor mehr nachweisbar ist, auf jegliche Operation verzichtet werden kann. Allerdings muss der Patient in diesen Fällen über die noch unzureichende Validierung dieses Vorgehens aufgeklärt werden und bereit sein, sich einer engmaschigen Nachsorge zu unterziehen [8].

Beim Plattenepithelkarzinom des thorakalen Ösophagus zeigen vergleichende Untersuchungen zwischen operativem Vorgehen mit neoadjuvanter Radiochemotherapie und der definitiven Radiochemotherapie keinen Unterschied im Überleben. Auch beim Ösophaguskarzinom kann es nach neoadjuvant durchgeführter Radiochemotherapie zu einer Komplettremission des Tumors kommen, sodass sich auch bei dieser Entität die Frage stellt, ob in diesen Fällen ein organerhaltendes Verfahren möglich und ein operativer chirurgischer Eingriff überhaupt noch notwendig ist. In der aktuell gültigen Version der S3-Leitlinie zur Diagnostik und Therapie der Plattenepithelkarzinome und Adenokarzinome des Ösophagus wird festgehalten, dass bei Patienten mit resektablen Plattenepithelkarzinomen des 
intrathorakalen Ösophagus der Kategorie cT3/cT4 alternativ zur chirurgischen Resektion auch eine definitive Radiochemotherapie durchgeführt werden kann [7]. Auch bei diesem Vorgehen wird auf eine Operation vollständig verzichtet und ein Organerhalt angestrebt. Auch nach neoadjuvanter Chemotherapie des Magenkarzinoms kann es zu Komplettremissionen mit der Unmöglichkeit eines Tumornachweises kommen.

\section{॥ Noch ist die sichere Feststellung einer kompletten Response schwierig}

Eine Vielzahl von Fragestellungen im Zusammenhang mit einer "complete response" nach neoadjuvanter Behandlung gastrointestinaler Karzinome ist jedoch zurzeit noch ungeklärt. Insbesondere die sichere Feststellung einer kompletten Response ist schwierig, da keines der zur Verfügung stehenden bildgebenden Verfahren eine sichere Aussage diesbezüglich möglich macht.

Nichtsdestotrotz wird der onkologisch tätige Viszeralchirurg zunehmend häufiger mit der Frage konfrontiert sein, ob er bei fehlendem Tumornachweis nach neoadjuvanter Vorbehandlung eines gastrointestinalen Karzinoms noch einen operativen Eingriff vornehmen will und soll.

Es ist das Ziel des vorliegenden Schwerpunkthefts der Zeitschrift Der Chirurg einen aktuellen Überblick zu dem Thema komplette Response nach neoadjuvanter Therapie gastrointestinaler Tumoren zu geben.

Die Arbeitsgruppe von Frau Tannapfel aus Bochum beschäftigt sich in ihrem Beitrag mit der Fragestellung, wie sicher die Pathologie in der Responsebeurteilung von Malignomen nach neoadjuvanter Therapie ist. Eisenblätter et al. aus Freiburg haben sich mit dem Thema der Diagnosestellung einer kompletten Response nach neoadjuvanter Therapie mithilfe radiologisch bildgebender Verfahren auseinandergesetzt. In den anschließenden Beiträgen werden die Implikationen für die Chirurgie nach kompletter Response beim Ösophaguskarzinom (Höppner, Lübeck), beim Magenkarzinom (Capovilla et al., Mainz) und beim Rektumkarzinom (Kastner et al., Würzburg) abgehandelt.
Wir hoffen mit diesem Schwerpunktheft der Zeitschrift Der Chirurg allen onkologisch tätigen Viszeralchirurgen einen aktuellen Überblick zum Thema Komplettremission nach neoadjuvanter Therapie solider Tumoren des Gastrointestinaltraktes in die Hand gegeben zu haben, der bei der täglichen Zusammenarbeit mit den Behandlungspartnern in den gastrointestinalen Tumorboards hilfreich ist.

Korrespondenzadresse

Univ.-Prof. Dr. med. C.-T. Germer

Klinik für Allgemein-, Viszeral-, Transplantations-, Gefäß- und Kinderchirurgie, Universitätsklinikum Würzburg Oberdürrbacher Str. 6, 97080 Würzburg, Deutschland germer_c@ukw.de

Interessenkonflikt. C.-T. Germer gibt an, dass kein Interessenkonflikt besteht.

\section{Literatur}

1. Babic B, Fuchs HF, Bruns CJ (2020) Neoadjuvante Radiochemotherapie oder Chemotherapie beim lokal fortgeschrittenen Ösophaguskarzinom? Chirurg 91:379-383

2. Bahadoer RR, Dijkstra EA, van Etten $B$ et al (2021) Short-course radiotherapy followed by chemotherapy before total mesorectal excision (TME) versus preoperative chemoradiotherapy, TME, and optional adjuvant chemotherapy in locally advanced rectal cancer (RAPIDO): a randomised, open-label, phase 3 trial. Lancet Oncol 22(1):29-42

3. Conroy T, Bosset JF, Etienne PL et al (2021) Neoadjuvant chemotherapy with FOLFIRINOX and preoperative chemoradiotherapy for patients with locally advanced rectal cancer (UNICANCERPRODIGE 23): a multicentre, randomised, openlabel, phase 3 trial. Lancet Oncol 22(5):702-715

4. Germer CT (2020) Neoadjuvante Konzepte in der chirurgischen Onkologie. Chirurg 91:377-378

5. Gockel I, Lordick F (2020) Neoadjuvante Chemotherapie beim Magenkarzinom. Vielfach eine Übertherapie oder ein sinnvolles Konzept? Chirurg 91:384-390

6. Kastner C, Petritsch B, Kim M, Germer CT, Wiegering $A$ (2020) Selektionskriterien zur neoadjuvanten Radiochemotherapie beim Rektumkarzinom. Chirurg 91:405-412

7. Porschen R, Fischbach W, Gockel I et al (2019) S3-Leitlinie - Diagnostik und Therapie der Plattenepithelkarzinome und Adenokarzinome des Ösophagus.Z Gastroenterol 57(3):336-418

8. Pox C, Aretz S, Bischoff SC et al (2013) S3-guideline colorectal cancer version 1.0. Z Gastroenterol 51(8):753-854

9. ScheufeleF, Friess H (2020) Neoadjuvante Therapie beim primär resektablen und Borderline-resektablen Pankreaskarzinom. Chirurg 91:391-395
Hier steht eine Anzeige. Springer 